American Journal of Applied Sciences 5 (11): 1562-1565, 2008

ISSN 1546-9239

(C) 2008 Science Publications

\title{
Image Compression and Reconstruction using Cubic Spline Interpolation Technique
}

\author{
${ }^{1}$ R. Muthaiah, ${ }^{2}$ K. NeelaKantan, ${ }^{3}$ Vidur Sharma and ${ }^{4}$ Amit Arora \\ ${ }^{1}$ TIFAC-CORE, Sastra University, Thanjavur, India \\ ${ }^{2}$ Consultant, NEC Electronics Singapore PTE, Ltd, Thailand \\ ${ }^{3}$ Department of Biotechnology, Sastra University, Inia \\ ${ }^{4}$ Department of Biotechnology, Sastra University, India
}

\begin{abstract}
A new dimension of image compression using random pixels of irregular sampling and image reconstruction using cubic-spline interpolation techniques proposed in this paper. It also covers the wide field of multimedia communication concerned with multimedia messaging (MMS) and image transfer through mobile phones and tried to find a mechanism to transfer images with minimum bandwidth requirement. This method would provide a better efficiency both in pixel reconstruction and color reproduction. The discussion covers theoretical techniques of random pixel selection, transfer and implementation of efficient reconstruction with cubic spline interpolation.
\end{abstract}

Key words: Random pixel selection, cubic-spline interpolation, transmission threshold, gray levels, runge phenomenon

\section{INTRODUCTION}

The high demand in multimedia and telecommunication industry for an efficient image compression technique emerged the development of this algorithm. The poor performance of H.26L at lower bit rates and the low quality color reproduction due to lesser bits allocated to chrominance is a real annoyance. The idea of taking random samples (pixels) from a picture and sending the randomization seed ${ }^{[1]}$ along with the random pixel set from a communication channel is exiting as well as efficient method in theory but its correct reconstruction poses a practical difficulty of crossing the Nyquist sampling barrier.

The main objective of this work to find a new compression method using random pixels sampled irregularly independent of the Nyquist rate and an efficient technique for image reconstruction instead of conventionally used iterative methods.

\section{RELATED WORKS AND METHODS}

This algorithm can be extended to moving pictures compression standards. Currently this has been implemented with static pictures and results also discussed in the later sections.

Random pixel selection: The process of random pixel selection for transfer depends on image quality that is being transferred. Here assumption is made that the gray levels of the image are uniformly distributed between 0 and 1 . That is the gray levels are normalized to be adjusted within the levels 0 and $1^{[1,2]}$. Then we try to generate a random number between 0 and 1 , we name it as "transmission threshold". Once the threshold is generated we scan the image to select pixel values that are above this threshold value. On the whole the algorithm for random pixel selection will be as follows:

- Normalize the gray levels in the image to range 0 and 1

- Now, select a random number between 0 and 1, name it the transmission threshold

- Scan the image to select all the pixels above this threshold value

- Transmit these pixels instead of entire image information

Since some of the pixel information is completely ignored it can be categorized as lossy compression technique.

Constraints to be checked: The threshold value must be suitable to transfer sufficient amount of information about the image so that, a picture with considerable clarity can be reconstructed.

- Also we should consider the losses that occur during the transmission of image. (That's why threshold is named transmission threshold)

Corresponding Author: R. Muthaiah, TIFAC-CORE, Sastra University, Thanjavur, India 
- If we are able to select such a threshold value, we can transmit sufficient amount of information that image of considerable quality can be reconstructed

Reconstruction: Reconstruction is an important stage in this process because it has to very efficient to extract maximum information from the given information. With such small amount of information being sent it necessary to have the best reconstruction technique to be used at the receiving end ${ }^{[3,4,5]}$. As we are sending only random information interpolation techniques can be utilized to reconstruct all the missing pixels in the image. Study of various interpolation techniques used for image reconstruction tells that the error in the interpolated value is more for most of techniques. Best of the choice will either polynomial interpolation or spline interpolation. Due to the effect of Runge's phenomenon on higher degree polynomial interpolation and computational complexity we choose spline interpolation as the best choice for reconstruction.

Spline interpolation: The existing techniques being not so consistent either with the efficiency or the speed or both, we try to get to the apotheosis of the reconstruction to be accomplished by using Cubicspline interpolation technique. A cubic-spline is a spline constructed of piecewise third-order polynomials which pass through a set of $m$ number of control points. The second derivative of each polynomial is commonly set to zero at the endpoints, since this provides a boundary condition that completes the system of $\left(\mathrm{m}^{2}\right)$ number of equations ${ }^{[6,7]}$. This produces a so-called natural cubic spline and leads to a simple tridiagonal system which can be solved easily to give the coefficients of the polynomials. However, this choice is not the only possible one, and other boundary conditions can be used instead following is the curve of how cubic spine looks like:

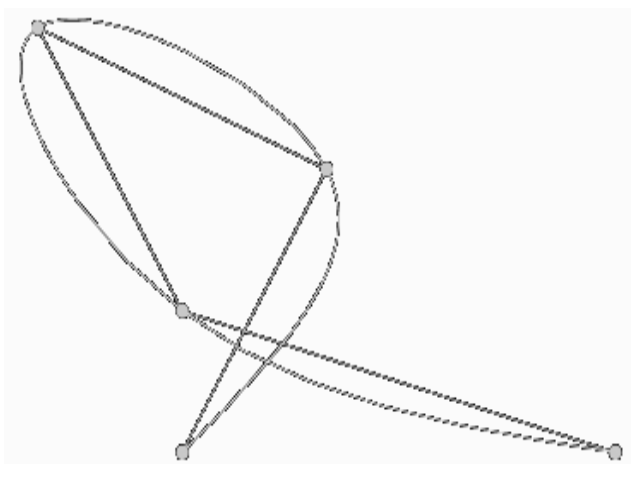

Cubic spline strategy in our context
As an interpolation method, this method also tends to derive the unknown values with the help of the known ones and tries to interpolate the values as closer to the original ones as possible. No mater what is the compression ratio of the signal is, the working of this interpolation (i.e., regeneration of the original signal from the limited information about it) remains same and thus, we have same set of equations governing this independent of the compression ratio, bandwidth, and other parameters of the signal. The main equation that explains its working is as follows:

For a data set $\left\{x_{i}\right\}$ of $n+1$ points, we can construct a cubic spline with $\mathrm{n}$ piecewise cubic_polynomials between the data points. If

$$
S(x)=\left\{\begin{array}{l}
S_{0}(x), x \in\left[x_{0}, x_{1}\right) \\
S_{1}(x), x \in\left[x_{1}, x_{2}\right) \\
S_{n-1}(x), x \in\left[x_{n-1}, x_{n}\right.
\end{array}\right.
$$

represents the splines function interpolating the function $\mathrm{f}$, we require, the interpolating property, $\mathrm{S}\left(\mathrm{x}_{\mathrm{i}}\right)$ $=\mathrm{f}\left(\mathrm{x}_{\mathrm{i}}\right)$, the splines to join up, $\mathrm{S}_{\mathrm{i}-1}\left(\mathrm{x}_{\mathrm{i}}\right)=\mathrm{S}_{\mathrm{i}}\left(\mathrm{x}_{\mathrm{i}}\right), \mathrm{i}=1, \ldots, \mathrm{n}-$ 1 twice continuous differentiable, $\mathrm{S}_{\mathrm{i}-1}^{\prime}\left(\mathrm{x}_{\mathrm{i}}\right)=\mathrm{S}_{\mathrm{i}}^{\prime}\left(\mathrm{x}_{\mathrm{i}}\right)$ and $\mathrm{S}_{\mathrm{i}-1}^{\prime \prime}\left(\mathrm{x}_{\mathrm{i}}\right)=\mathrm{S}_{\mathrm{i}}^{\prime \prime}\left(\mathrm{x}_{\mathrm{i}}\right), \mathrm{i}=1, \ldots, \mathrm{n}-1$.

For the $\mathrm{n}$ cubic polynomials comprising $\mathrm{S}$, this means to determine these polynomials, we need to determine $4 \mathrm{n}$ conditions (since for one polynomial of degree three, there are four conditions on choosing the curve $)^{[8]}$. However, the interpolating property gives us $\mathrm{n}+1$ conditions, and the conditions on the interior data points give us $n+1-2=n-1$ data points each, summing to $4 \mathrm{n}-2$ conditions. We require two other conditions, and these can be imposed upon the problem for different reasons.

As we can very well infer from the above equation that the unknown variables can be easily deduced from the very few known variables. As compared to the original values we observed that the error has been very low particularly as far as pixel loss and color loss are concerned. Thus this is very obvious to conclude that this method is most suitable in this context in present scenario.

Advantages of Cubic-spline: Now this is a very genuine question to be provoked here that why do we go for cubic-spline or how this method is superior to others, so here is the answer. In some situations, interpolating polynomials are not suitable since they exhibit an oscillating behavior as their degree increases.

To overcome this oscillatory behavior and still provide a smooth interpolation, we have used cubicspline interpolation technique on neighboring integer 
located intensities to obtain the null interpolated intensity for that sub pixel location.

Secondly, from the knowledge of other interpolating methods, we infer that either they solve the matrix elements either row wise or column wise. But if we see the cubic-spline curve, its obvious that it covers a 2-D area with just two points. Then with the next set of interpolation points, the previously calculated values are rectified tending to minimum error.

The idea here is that since the neighborhood displacements are usually similar while neighboring intensities may be less. So, for this, we found cubicspline interpolation to be more accurate than other existing techniques like, for instance, linear patch interpolation uses 4 sub pixel locations in its calculation while cubic-spline interpolation uses 25 sub pixel locations in its calculations, hence improved efficiency is obvious.

\section{RESULTS AND DISCUSSION}

Application of cubic-spline interpolation technique for reconstruction of image from irregular samples by applying the proposed construction technique, we have achieved the following results of Lena images from Fig. 1 and 2 of scenery image.

Error estimation in reconstruction-tabulation statistics: Irregular reconstruction is a lossy technique and its efficiency depends on its accuracy. By taking $10 \%$ of the pixels randomly we have achieved $81.52 \%$ accurate reconstruction of the original Lena picture. In general reconstructed images on comparison with the original image give quite an acceptable error percentage both in pixels loss and the color loss. Pixel loss is calculated by matching the original pixel value to the reconstructed one by having a threshold of 0.1 , the same threshold is used of calculating color loss. Table 1 shows the error in Lena image reconstruction.

This error estimation table is obtained by the help of the software named image diffing whose copyright is with the ionforge company.

Referring to the above statistics obtained based on the proposed theory, that can assure to achieve a good compression ratio provide satisfactorily high quality reconstruction. The results depict the high level of efficiency of the reconstruction algorithms and the superiority of irregular sampling in this area. By the virtue of these methods, the results obtained $90 \%$ compression ratio with $18 \%$ pixel loss and $2 \%$ color loss which is acceptable in conditions where high resolution images are to be sent from a low band-width

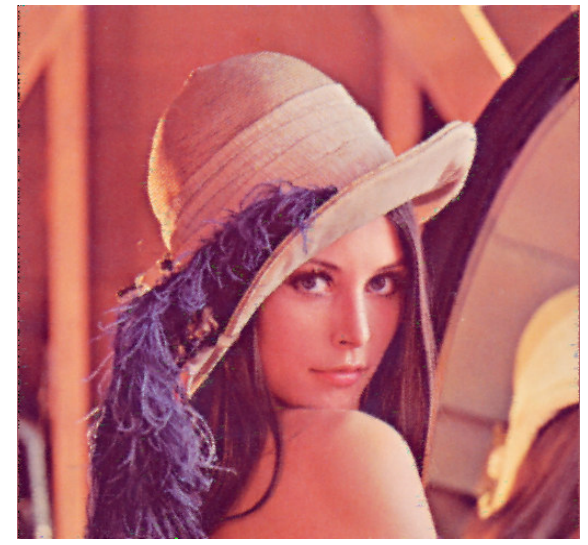

Fig. 1a: Original Lena

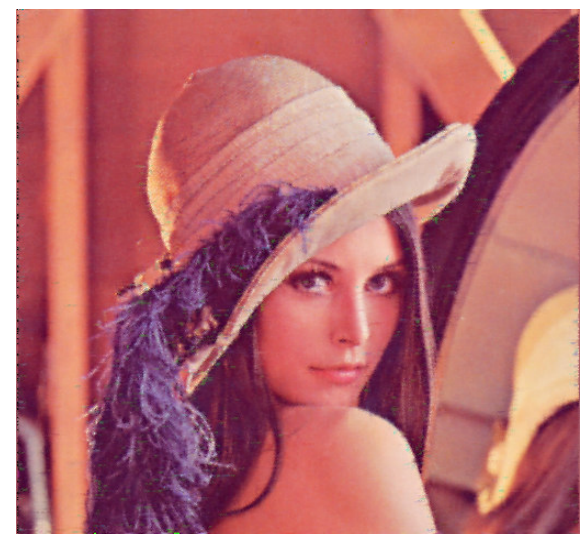

Fig. 1b: Reconstruction with 50\% pixels taken

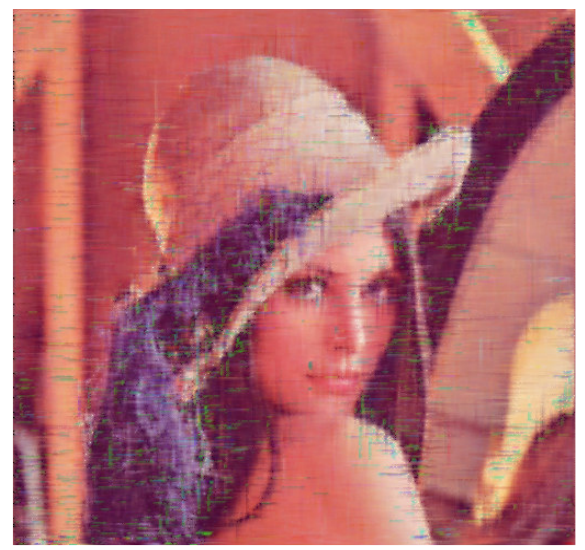

Fig. 1c: Reconstruction with 10\% pixels taken

channel (like mobile communication channels). Obviously, the technique of cubic-spline interpolation is really very impressive to improve the efficiency of compression. 


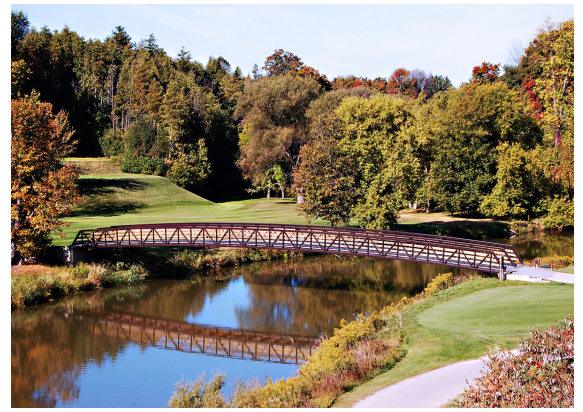

Fig. 2a: Original scenery image

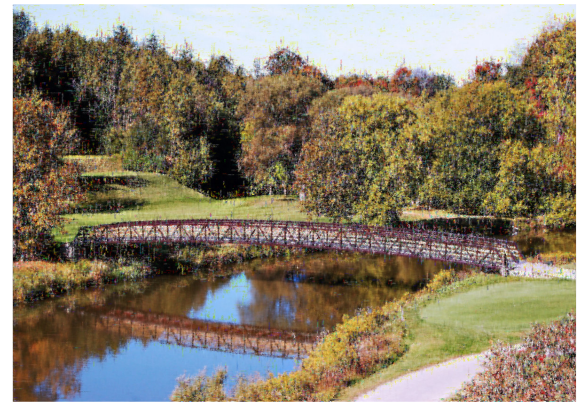

Fig. 2b: Reconstruction with $15 \%$ pixels taken

Table 1: For Lena image

\begin{tabular}{lll}
\hline Pixels used for reconstruction $(\%)$ & Pixel loss $(\%)$ & Color loss $(\%)$ \\
\hline 50 & 2.17 & 0.23 \\
40 & 3.19 & 0.34 \\
30 & 4.95 & 0.53 \\
420 & 8.54 & 0.94 \\
10 & 18.48 & 2.13 \\
\hline
\end{tabular}

\section{REFERENCES}

1. Le Foch, H. and C. Labit, 1996. Irregular image sub-sampling and reconstruction by adaptive subsampling. Proceedings of ICIP'96, vol. III, Lausanne.

2. Ramponi, G. and S. Carrato, 2001. An adaptive irregular sampling algorithm and its application to image coding. Image Vision Comput., vol. 19.

3. Yeh, S. and H. Stark, 1990. Iterative and one-step reconstruction from nonuniform samples by convex projections. J. Opt. Soc. Am. A. Opt. Image Sci., 7 (3): 491-499.

4. Marvasti, F., 1996. Interpolation of Lowpass signals at half the Nyquist Rate IEEE. Sigmal Processing Letters, Vol. 3.

5. Marvasti, F., M. Analoui and M. Gamshadzahi, 1991. Recovery of signals from nonuniform samples using iterative methods IEEE. Transactions in Signal Processing, 39 (4).

6. Ken D. Sauer and P. Jan Allebach, 1987. Iterative reconstruction of band-limited images from nonuniformly spaced samples, IEEE. Transa. Circuits Systems. Vol. 34.

7. Feichtinger, H.G. and T. Strohmer, 1993. Fast iterative reconstruction of band-limited images from irregular sampling values. Comput. Anal. Images Patterns, pp: 82-91.

8. Aldroubi, A. and K. Groechening, 2001. Nonuniform sampling and reconstruction in shiftinvariant spaces. SIAM Review. 